\title{
Multiplicity of Terms Related to Patient Safety: A Hindrance to Progress
}

\author{
Marc Perreault
}

Co many terms related to patient safety, yet so little progress in $\checkmark$ this area over the years! Patient safety has become a major endeavour within virtually every health care setting, yet despite the efforts of every health care professional, patients continue to be at risk of adverse events as a result of direct care and processes of care.

A new term is now starting to appear in the literature: "patient safety incident". This term originates from the World Health Organization (WHO), which in 2004 set up the WHO Patient Safety program and the International Classification for Patient Safety in a global effort to standardize definitions, classify risks, and develop and compare strategies to improve patient safety. ${ }^{1}$ A patient safety incident is defined as "an event or circumstance that could have resulted, or did result, in unnecessary harm to a patient." 2 The term "patient safety incident" — along with 47 others, such as "harmful incident", "no harm incident", and "near miss" - covers the entire range of patient safety within health care, not exclusively medications. This terminology arises from a move toward a culture of patient safety that aims to avoid blame and retribution and to promote learning and improvement within health care institutions. Has the WHO succeeded in achieving this goal over the past 10 years? I don't think so.

As workers in the health care system, we encounter difficulties in interpreting various terms and their definitions as used within the realm of patient safety. These terms are often understood very differently depending on the individuals or professionals involved; their meaning also depends on the domain of use, whether this be clinical, regulatory, or even legal. ${ }^{3}$ In addition, if the focus is on medication-related terms, confusion may arise from whether one is referring to the process of medication use, to the clinical outcomes of medication use, or to a combination of process and outcome. $^{3}$

As a pharmacist, I am first and foremost concerned with safety issues arising from medications that are given to my patients. Both proper and improper uses of medications have the potential to create significant burdens on the health care system, because of a patient's admission to hospital from a drug-related problem, increased length of stay, or increased morbidity and mortality. For example, use of second-generation antipsychotics is increasing for a variety of psychiatric conditions, including schizophrenia and critical illness delirium, and such wider

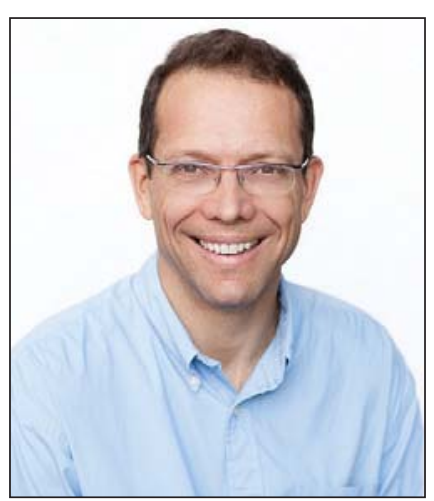
use leads to the observation of rare adverse drug reactions, such as clozapine-induced myocarditis. This condition is the focus of a case report by Barry and others ${ }^{4}$ appearing elsewhere in this issue. These authors refer to the problem as both an adverse drug reaction and an adverse event, whereas other health care professionals might describe it as an adverse drug effect or an adverse drug event. Such terms are often used interchangeably, despite their slightly different meanings, which renders comparisons among studies difficult; estimating with precision the magnitude of the real problem becomes impossible. ${ }^{5,6}$ In an extensive review of the medication-related terms and definitions used in patient safety, Pintor-Mármol and others ${ }^{3}$ identified a total of 60 terms used, with 189 different definitions. This heterogeneity of terms and definitions significantly hinders advancement in the field of patient safety.

Now, to what extent has the term "patient safety incident" been adopted in the literature since 2004? Although I concur with the WHO's global objectives, the term has remained mostly theoretical and has not yet gained widespread acceptance or buyin from the health care community. Studies performed in England applied this terminology to the fields of hospital deaths ${ }^{7}$ and cardiac arrest, ${ }^{8}$ but overall, adoption has been limited. For consistency, the Canadian Patient Safety Institute (CPSI) 
preferred the WHO term "patient safety incident" in its Canadian Incident Analysis Framework, published in 2012; however, the CPSI recognized that organizations might have reasons to continue using other terminology. ${ }^{9}$ For the term "patient safety incident" to become widely accepted in Canada, we need to agree on a set of terms (including this one), a timeline for implementation, and commitment and support from organizations nationwide.

In our ongoing efforts to make our health care system safer, we all recognize the need to identify unifying terms, which might include "patient safety incident" or others, with clear definitions and concepts. At all levels (national, provincial, and local), we will need to adopt the same terminology so it can be applied uniformly across disciplines. Until we truly embrace the patient safety culture and move to a system that universally reports all incidents, health care professionals will not be motivated to report and will fail to explore new ways of improving patient safety and overall care. ${ }^{10}$ And until these difficulties are overcome, we will unfortunately continue to face a multiplicity of terms and definitions and therefore may underestimate the safety incidents that our patients experience.

\footnotetext{
References

1. Donaldson SL. An international language for patient safety: global progress in patient safety requires classification of key concepts. Int J Qual Health Care. 2009;21(1):1.

2. Runciman W, Hibbert P, Thomson R, Van Der Schaaf T, Sherman H, Lewalle P. Towards an international classification for patient safety: key concepts and terms. Int J Qual Health Care. 2009;21(1):18-26.

3. Pintor-Mármol A, Baena MI, Fajardo PC, Sabater-Hernández D, SáezBenito L, García-Cárdenas MV, et al. Terms used in patient safety related to medication: a literature review. Pharmacoepidemiol Drug Saf. 2012; 21(8):799-809.
}

4. Barry AR, Windram JD,Graham MM. Clozapine-associated myocarditis: case report and review of the literature. Can J Hosp Pharm. 2015;68(5): 427-9.

5. Aronson JK. Distinguishing hazards and harms, adverse drug effects and adverse drug reactions: implications for drug development, clinical trials, pharmacovigilance, biomarkers, and monitoring. Drug Saf. 2013;36(3): 147-53.

6. Runciman WB. Shared meanings: preferred terms and definitions for safety and quality concepts. Med J Aust. 2006;184(10 Suppl):S41-3.

7. Donaldson LJ, Panesar SS, Darzi A. Patient-safety-related hospital deaths in England: thematic analysis of incidents reported to a national database, 2010-2012. PLOS Med. 2014;11(6):e1001667.

8. Panesar SS, Ignatowicz AM, Donaldson LJ. Errors in the management of cardiac arrests: an observational study of patient safety incidents in England. Resuscitation. 2014;85(12):1759-63.

9. Canadian Patient Safety Institute (CPSI); ISMP Canada; Saskatchewan Health; Patients for Patient Safety Canada; Beard P. Hoffman CE, Ste-Marie M. Canadian incident analysis framework. Edmonton (AB): CPSI; 2012 [cited 2015 Jul 25]. Available from: www.patientsafetyinstitute.ca/en/ toolsResources/IncidentAnalysis/Documents/Canadian\%20Incident\% 20Analysis\%20Framework.PDF

10. Larizgoitia I, Bouesseau MC, Kelley E. WHO efforts to promote reporting of adverse events and global learning. J Public Health Res. 2013;2(3):e29.

Marc Perreault, BPharm, MSc, PharmD, is a Critical Care Pharmacist at the Montreal General Hospital, Montréal, Quebec. He is also an Associate Editor with the CJHP.

Competing interests: None declared.

\section{Address correspondence to:}

Dr Marc Perreault

Pharmacy Department

Montreal General Hospital

1650 Cedar Avenue, Suite C1-200

Montreal QC H3G 1A4

e-mail : marc.perreault@umontreal.ca

\section{ON THE FRONT COVER}

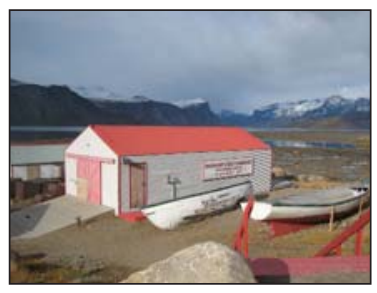

\section{Pangnirtung, Nunavut}

The Hudson's Bay Company blubber processing plant (circa 1921) is seen against the backdrop of the Pangnirtung Fiord. Located $40 \mathrm{~km}$ south of the Arctic Circle on Baffin Island, Pangnirtung is a small hamlet (population 1325) famous for its tapestries, prints, and "Pang" hats. Heather Kertland,
Clinical Pharmacy Specialist/Leader at St Michael's Hospital, Toronto, Ontario, took this photo during a visit over Labour Day weekend a few years ago. The first snow of the season had fallen the previous night and can still be seen on the higher elevations of land.

The CJHP would be pleased to consider photographs featuring Canadian scenery taken by CSHP members for use on the front cover of the journal. If you would like to submit a photograph, please send an electronic copy (minimum resolution $300 \mathrm{dpi}$ ) to cjhpedit@cshp.ca. 